\title{
Foreword from the editors...
}

\author{
Marc Hallin $^{1}$. Yury Kutoyants ${ }^{2}$
}

Published online: 15 June 2018

(C) Springer Nature B.V. 2018

The first volume of Statistical Inference for Stochastic Processes appeared 20 years ago. Launched as a Kluwer journal, it was rebranded as a Springer publication after the merger of Kluwer Academic Publishers and Springer.

The first issue, dated January 1998, contained a Foreword by its founding Editor-in-Chief Denis Bosq, at that time Director of the Institut de Statistique de l'Universite de Paris, and the volume featured a total of 16 papers, several of them by current Associate Editors of the journal: Serge Dachian, Christian Francq, Marc Hallin, Reinhard Höpfner, Yury Kutoyants, Serguey Pergamenshchikov, Nakahiro Yoshida. Since then, 60 issues have appeared, with a total of 346 articles.

The journal was born from the growing feeling, in the mid-nineties, that the statistical analysis of continuous-time processes, as well as that of functional observations, were in need of a specific publication venue. "Traditional" journals indeed had a tendency to reject submissions in these areas on the pretext that time and the observations anyway always are discrete in practice, so that continuous time and functional models had little connection to reality. On the other hand, restricting to continuous time overly narrows the scope, as discrete- and continuous-time are intimately intermingled (cfr the AR(1) simulation of diffusions, or the AR(1) Euler discretization scheme of Ornstein-Uhlenbeck processes). The idea thus slowly emerged of a journal where those two branches of statistics- the statistics of continuoustime and the analysis of time series-would live under the same roof. And, in 1998, the journal was launched as a regular Kluwer publication, accepting submissions on all subjects

Marc Hallin

mhallin@ulb.ac.be

$\bowtie$ Yury Kutoyants

Yury.Kutoyants@univ-lemans.fr

1 Université libre de Bruxelles, Brussels, Belgium

2 Université du Maine, Le Mans, France 
dealing with, or related to, the statistical analysis of dependent observations-continuous- or discrete-time, spatial or spatio-temporal, univariate, multivariate, or functional observations.

The field of continuous-time statistics since then has been developing at a rapid pace, though. Two series of workshops, in particular, were started in the late nineties — one, "Asymptotic Statistics of Stochastic Processes", organized every second year since 1997 in Le Mans by Yury Kutoyants and Nakahiro Yoshida, another one, conveyed on a yearly basis since 1994 by Michael Sørensen (under the name DYNSTOCH from 2000 on). Those workshops have attracted many young statisticians to the subject, and the journal has become home to many of them.

The overall situation has evolved, too, over the past 20 years, and the topics Statistical Inference for Stochastic Processes was created for nowadays are receiving much better recognition from the statistical community. We like to think that our journal had some influence on that evolution.

To celebrate this 20th anniversary, Springer accepted the idea of a special 20th anniversary issue by which members of the Editorial Board reaffirm their commitment to the journal by submitting a contribution. We take this opportunity to thank, very warmly, all those who- publishers, editors, authors, referees - made those 20 years an increasing success and, hopefully, will keep on supporting us for the many years to come.

Above all, we should like to express our very special gratitude to Denis Bosq and the members of the 1998 editorial board, who have been running the editorial process in the early years, and settled the journal's high scientific standards.

Marc Hallin and Yury Kutoyants

April 2018

\section{... and from the founding editor}

It gives me real pleasure to see this special 20th anniversary issue in print, and to be able to contribute to it. My warmest thanks go to Yury Kutoyants, who faithfully stood by my side from the very beginning of the project, and without the support of whom nothing would have been possible. I also feel quite grateful to the entire editorial board who helped me running the journal though all those years, and to Marc and Yury again, for taking over, in 2013, my editorial duties with competence and enthusiasm. Thanks to you, the journal is more vigorous and lively than ever, and a reference in the field.

Long life to Statistical Inference for Stochastic Processes!

Denis Bosq

May 2018 